\title{
THE USE OF BIOPOLICIDE FOR PROTECTIVE-STIMULATING MIXTURES
}

\author{
A. M. Klymenko, Ya. V. Chabaniuk \\ Institute of Agroecology and Environmental Management, NAAS, Kyiv \\ 12, Metrolohichna Str., Kyiv City, 03143, Ukraine e-mail: alinank@ukr.net
}

One of the important elements of the process of growing crops, which affects the increase of productivity and quality of crop production, is preplant seed treatment. In modern agronomic practice the use of chemical seed disinfectant before sowing is deemed a necessary measure to improve seeding material. However, along with the achievement of positive results, the use of crop protection chemicals has several negative consequences, including the pollution of environment with pollutants, accumulation of dangerous chemicals both in the soil and in crop production.

Seeds, entering the market of Ukraine, are usually disinfected on foreign seed plants by incrustation to increase its sowing qualities. Domestic seeding material often does not meet the increasing demands of safety and quality, so the purchase of quality seeding material is always important for agricultural producers.

Incrustation is seen as guaranteed factor of increasing yields by protecting seeds from bacterial, fungal and viral diseases that exist in soil, on seeds or inside it from pests, which are in the soil, and by stimulating the growth process [1]. In recent years, the impact of various factors of the environment on quality characteristics of seeds has been studied and methods for effective improvement of seeds stability against the negative impact of unfavourable soil factors were proposed. Among them particularly important are protective-stimulating mixtures (PSM), which form a protective film on seeds surface, which significantly increases the germinating energy, field seed germination, uniform growth and development of young plants, forming improved quality crops. Inlaid seeds can be sown 5-6 days before the usual time limits.

Incrustation with PSM allows to create a coloured shell around the seed with the required set of compounds, consisting of:
- composition of plant protection from diseases and pests;

- growth processes stimulators;

- film-forming substance;

- trace elements in chelated form;

- carrier, adsorbent;

- dye.

Element-material composition and balance between PSM individual components is formed on the basis of physiological requirements of crops and soil and climatic characteristics of their cultivation.

However, the system of corn incrustation needs to be improved, as the possibility of compatible application of several components to the surface of seeds, namely disinfectants, fertilizers and plant growth regulators has not yet been fully elucidated.

In connection with the prospects of this agricultural practice there occurred the necessity for the research of the effectiveness of preplant corn seed incrustation with the composition comprising microbial preparation Biopolicide. The effectiveness of Biopolicide against phytopathogenic bacteria and fungi was confirmed earlier [8].

The objective of this work was to study the possibility of creating protectivestimulating mixtures based on Biopolicide for preplant treatment of corn seed.

Materials and methods. Biopolicide preparation is based on spore-forming bacteria Paenibacillus polymyxa $6 \mathrm{M}$ with the complex of agronomically useful properties. The separation of bacilli spores from culture fluid to create a concentrated biological preparation was performed by centrifugation.

To study Biopolicide compatibility with major insecticidal substances preparations Gaucho and Cruiser 600 FS were selected.

Gaucho is a disinfectant of systemic and contact action against a broad spectrum of pests. Active substance of the preparation 
(imidacloprid) belongs to a new group of active ingredients - chloronicotiniles.

Cruiser 600 FS is a systemic insecticide for preplant seed treatment against a wide range of pests. Active substance of the preparation - thiamethoxam.

The following colours were used in the experiment: karamzin, betanin, indigo, copper complex of chlorophyll.

The evaluation of the cytotoxic action of insecticides and dyes was performed by paper disk method [7].

Mathematical processing of results was carried out by conventional methods [2].
Therefore, seed treatment with the mixture containing water-soluble protective film, disinfectant and micronutrients in its composition is a rather effective way of preplant seed preparation, which allows to fix fast all of the above components on the surface of seeds with plastic film. We found the possibility of using Biopolicide as a part of protective-stimulating compositions for the treatment of corn seed. The findings suggest that biological agents are resistant to studied chemical insecticides, polymeric compounds and dyes, which gives reason to create a number of combinations depending on the culture needs and soil and climatic conditions. 